\title{
The fermentation characteristics of Sparassis crispa polysaccharides and their effects on the intestinal microbes in mice
}

\author{
Meng-yang Liu' ${ }^{1}$, Shao-jun Yun ${ }^{1}$, Jin-ling Cao ${ }^{1}$, Feier Cheng ${ }^{1}$, Ming-chang Chang ${ }^{1,2}$, Jun-long Meng ${ }^{1,2}$, \\ Jing-yu Liu ${ }^{1}$, Yan-fen Cheng ${ }^{1}$, Li-jing X ${ }^{1}{ }^{1}$, Xue-ran Geng ${ }^{1}$ and Cui-ping Feng ${ }^{{ }^{*}}$
}

\begin{abstract}
Background: Sparassis crispa polysaccharides (SCPS) have multiple pharmacological activities. Fermentation characteristics of SCPs and its effects on the intestinal microbes in mice remain inconclusive.

Results: In this study, SCPs were fermented by the human feces and used to administer the Kunming mice to explore the fermentation characteristics of SCPs in the intestinal tract and the effects on the intestinal microbes in mice. Results from in vitro experiments revealed that SCPs were utilized by intestinal microbiota to produce short-chain fatty acids (SCFAs). The specific monosaccharide composition of SCPs determines which SCFAs are produced. Furthermore, the colon index and villi length of the SCPs-treated mice were significantly higher compared with the control group. In addition, SCPs exhibited beneficial effect on the relative abundance and diversity of dominant bacteria in the intestinal tract, such as increasing Bacteroidetes/Firmicutes ratio and up-regulating SCFA-producing bacteria, including Bacteroidales_S24-7_group, Alloprevotella, Alistipes, Bacteroides, Butyricimonas, Parabacteroides, Lachnospiraceae_NK4A136_group and Oscillibacter. SCPs increased the abundance of genes in carbohydrate, amino acid, and energy metabolism.

Conclusion: Our results indicate SCPs can improve the physiological indices of the colon in mice, which is likely to be associated with the increase in the relative abundance and diversity of SCFA-producing bacteria and SCFAs level produced by intestinal microbiota.
\end{abstract}

Keywords: Sparassis crispa polysaccharides, Short-chain fatty acids, Colon, $16 \mathrm{~S}$ rDNA high-throughput sequencing, Relative abundance

\section{Introduction}

Intestinal microecosystems, composed of intestinal microbiota and the environment in which they live, are one of the most important and complex human microecosystems. Intestinal microbiota plays an important physiological role in host immunity and metabolism and is affected by internal and external environmental factors [1]. Moreover, an imbalance within the intestinal

\footnotetext{
*Correspondence: ndfcp@163.com

${ }^{1}$ College of Food Science and Engineering, Shanxi Agricultural University,

Taigu, Shanxi 030801, The People's Republic of China

Full list of author information is available at the end of the article
}

microecology is associated with the development of certain diseases [2]. Indeed, the reduction of intestinal microbiota diversity has been proved to induce obesity and low-level inflammation and changes in microbial flora composition are closely associated with the development of diabetes, coronary heart disease, and stroke [3]. Studies have shown that intestinal microbiota diversity and abundance can be regulated by adjusting our diets [4-7], such as taking more dietary polysaccharides, to maintain human health $[8,9]$. For example, gut metabolites of blackberry ameliorated ROS overproduction, mitochondrial membrane collapse, and glutathione 
depletion induced by high glucose plus palmitic acid in HepG2 cells [4]. Pelargonidin-3-O-glucoside not only modified the gut microbiota composition, but also strengthened the intestinal barrier integrity [8].

Polysaccharides play important roles in the maintenance of human health. Specifically, polysaccharides have defined functions in hypoglycemia, hyperlipidemia, immune regulation, intestinal defecation [10], antiinflammation [11], anti-oxidation [12], and protection of lipotoxicity [13] and the intestinal barrier structure and function $[14,15]$. For example, the polysaccharide from Rubus chingii $\mathrm{Hu}$ had the cytoprotective effect against palmitic acid-induced lipotoxicity in normal human hepatocyte cell line L02 [13]. Co-consumption of green tea derived products with wheat starch could delay the intestinal absorption of glucose [14]. Furthermore, although the human body is incapable of absorbing and digesting polysaccharides, they can be transferred to the large intestine where they are fermented by intestinal microorganisms to produce lactic acid, ethanol, succinic acid and other intermediates, and subsequently be converted into short-chain fatty acids (SCFAs) [16]. Recently, increasing attention has been given to study the effect of polysaccharides on intestinal function. The various polysaccharide structures, including chain length, types of glycoside bonds, connection modes, and molecular weight, affect the intestinal microflora composition [17, 18]. Specifically, polysaccharides from Helicteres angustifolia $L$ significantly elevated the relative abundance of Actinobacteria and the ratio of Bacteroidetes to Firmicutes [19]. Lentinula polysaccharides also significantly decreased the amount and the diversity of OTUs in gut microbiota [20]. Dendrobium officinale polysaccharides (DOP) increased the population of Firmicutes and Bacteroidetes and decreased the abundance of Proteobacteria [21]. Thus, most natural polysaccharides have a potential application as novel prebiotics by promoting the reproduction of Bifidobacterium or Lactobacillus and increasing the production of SCFAs.

Sparassis crispa (S. crispa) is a kind of rare, medicinal, edible mushroom with a fruiting body that has rich nutritional value containing many active substances. The main component of $S$. crispa is $\beta$-glucan, and its content is between 40 and $43 \%$, which is the highest among mushrooms [22]. S. crispa polysaccharides (SCPs) containing $\beta$-glucan are reported to possess anti-tumor effects in mice with strong vascular dilation and hemorrhage reactions [23]. Oral administration of $\beta$-glucan from S. crispa may modulate cytokine induction in the spleen through the activation of Peyer's patches [24]. It has been successfully confirmed that SCPs exhibit anti-allergic effect in both in vivo and in vitro models [25]. Purified SCPs exhibit neuro-protective activity against L-Glu-induced neuronal apoptosis in DPC12 cells and can be a potential therapeutic or modulating agent for neurodegenerative damage [26]. Furthermore, previous studies showed that the $\beta$-glucans in mushroom could not be digested in the human gastrointestinal tract and were thus considered as a potential source of prebiotics. Mushroom $\beta$-glucans possess profound health promoting properties like speeding up the transit of bowel contents, increasing fecal bulk and frequency, consequently protecting the body from colon cancer, diverticular diseases and irritable bowel syndrome [27]. Although many studies on the SCPs have been carried out, data on the fermentation characteristics of SCPs and their effects on the intestinal microbes in mice are limited and the underlying mechanism of the effects of SCPs on intestinal microbes are still largely unclear.

Thus, in this study, SCPs were fermented by the human feces to explore the fermentation characteristics of SCPs in the intestinal tract and used to administer the Kunming mice to investigate their effects on the intestinal microbes in mice. The results of this study may be useful for further applications of SCPs as a new hydrocolloid source in food industries.

\section{Materials and methods \\ Reagents}

Concentrated sulfuric acid $\left(\mathrm{H}_{2} \mathrm{SO}_{4}\right)$, 3,5-dinitrosalicylic acid (DNS), sodium hydroxide $(\mathrm{NaOH})$, and sodium sulfite $\left(\mathrm{Na}_{2} \mathrm{SO}_{3}\right)$ were analytical reagents and were obtained from Tianjing Chemical Reagent 3th Factory (Tianjing, China). Xylose, fructose, arabinose, mannose, glucose, galactose, and fructose were purchased from Solarbio Technology Co., Ltd (Solarbio, Beijing, China).

\section{SCPs extraction and identification}

S. crispa was supplied by the Edible Mushroom Center of Shanxi Agricultural University (Shanxi, China). The SCPs were prepared according to our previous method [28]. Briefly, S. crispa was ground into powder, passed through 200-mesh sieves, and macerated in distilled water $(1: 38.8[\mathrm{w} / \mathrm{v}])$ for $3 \mathrm{~h}$ at $80^{\circ} \mathrm{C}$. Samples were then centrifuged and added triploid volume of anhydrous ethanol overnight at $4{ }^{\circ} \mathrm{C}$ to precipitate the extract. Crude SCPs were separated from the protein using the savage method and purified by HZ830 macroporous resin and a diethylaminoethyl (DEAE)-cellulose column (Solarbio, Beijing, China). The estimated molecular weight of SCPs was $215 \mathrm{Da} \sim 393 \mathrm{kDa}$ by high-performance gel permeation chromatography analysis [28]. The monosaccharide compositions of SCPs were galactose, glucose, xylose, mannose, and fructose in the mole ratios of 10.55: 24.76: 2.51: 5.64: 1 using ion chromatography analysis following complete acid hydrolysis [28]. The major structural units 
were a $\beta$-( $1 \rightarrow 3)$-D-glucan with a single $\beta$ - $(1 \rightarrow 6)$-D-glucosyl side branching every three residues based on $1 \mathrm{D}$ and 2D NMR spectrum analysis [28].

\section{In vitro experiments}

\section{Preparation of human intestinal microbiota}

The fresh feces from six healthy adults (25 years old, 3 males and 3 females) who had no history of gastrointestinal diseases and had not used antibiotics within 3 months were collected and mixed thoroughly. Mixed solids were added to a Dulbecco's phosphate-buffered saline (DPBS, $10 \%[\mathrm{v} / \mathrm{v}])$ solution with a final concentration of $20 \%$ $(\mathrm{w} / \mathrm{w})$. After being homogenized for $1 \mathrm{~min}$, the homogenate was filtered with double nylon gauze, and the resulting liquid was separated into a sterilized $100 \mathrm{~mL}$ conical tube and packed with a stopper until further use. All study participants provided informed consent, and the study was approved by the Ethics Committee of Shanxi Agricultural University and carried out in accordance with Declaration of Helsinki.

\section{Detection of biochemical indexes in fermentation broth}

The prepared SCPs solution $(2 \mathrm{mg} / \mathrm{mL})$ was autoclaved, added to conical tubes containing human intestinal microbiota, and placed into aseptic anaerobic culture bags (Haibo, Qingdao, China). The bags were sealed and the tubes were shaken seven times to mix the contents evenly. Then the mixtures were fermented at $37^{\circ} \mathrm{C}$ for 1 , $2,6,12,24$, and $48 \mathrm{~h}$, respectively. Uncultured fermentation broth $(0 \mathrm{~h})$ was used as control. The $\mathrm{pH}$ of the fermentation broth was determined using a $\mathrm{pH}$ meter (ST3100; Ohaus Instruments, Parsippany, NJ, America) and the $\mathrm{OD}_{600}$ value was measured using an ultraviolet spectrophotometer (Cary 4000; Agilent, Santa Clara, $\mathrm{CA}$, America). The total sugar content in the fermentation broth was measured using the phenol-sulfuric acid method, and the reducing sugar content in the fermentation broth was determined by the DNS method $[29,30]$. The monosaccharide composition in the fermentation broth was assayed according to a previous method [31]. Briefly, clarified fermentation liquid was filtered with a $0.45 \mu \mathrm{m}$ water-based filter, collected in a $1.5 \mathrm{~mL}$ brown sample bottle, and determined by the Shimadzu 2014C gas chromatography (Agilent Technologies Inc., Santa Clara, America). The temperature of the injector and the detector were $250{ }^{\circ} \mathrm{C}$ and $280{ }^{\circ} \mathrm{C}$, respectively. The injection dose of $1 \mu \mathrm{L}$ was normal injection without splitting. The flow rate of carrier gas $\left(\mathrm{N}_{2}\right)$, air and hydrogen were $1.5,450$ and $60 \mathrm{~mL} / \mathrm{min}$, respectively. Finally, the SCFAs composition was assessed using solid-phase microextraction (SPME)-GC-MS (Thermo Fisher, Shanghai, China). An HP-INNOWAX $(30 \mathrm{~m} \times 0.32 \mathrm{~mm}$ ID) column was employed and the injection parameters for the GC-MS were as follows: helium as the carrier gas, flow rate of $19.0 \mathrm{~mL} / \mathrm{min}$, non-shunt injection and heating at $100^{\circ} \mathrm{C}$ for $30 \mathrm{~s}$, and a heating rate of $4{ }^{\circ} \mathrm{C} / \mathrm{min}$ from $100{ }^{\circ} \mathrm{C}$ to $180^{\circ} \mathrm{C}$.

\section{In vivo experiments \\ Experimental design}

Male Kunming mice ( 6 weeks old, $25 \pm 2$ g) were purchased from Weitong Lihua Animal Testing Co., Ltd. (Beijing, China) and placed in an animal house with the appropriate environmental condition (temperature of $22 \pm 0.5{ }^{\circ} \mathrm{C}$, humidity of $50 \pm 5 \%$, and a light: dark cycle of 12:12 h) for 7 days to acclimate the test conditions, during which they had free access to standard laboratory pellets and water. Experimental animal protocols and procedures complied with the Guide for the Care and Use of Laboratory Animals (U.S. National Academy of Sciences, National Institutes of Health Publication $6-23$, revised 1985) and the principles and procedures of the European Communities Council Directives (86/609/ EEC). The protocols also received an approval from the Ethics committee of Shanxi Agricultural University. Every effort was made to maximize the well-being of the mice, minimize their suffering and the number of animals needed for the study.

After the acclimation, 96 healthy male mice were randomly divided into four groups. The control group (CG) was administered intragastrically by $0.9 \%$ normal saline and the low-dose group (LDG), medium dose group (MDG) and high-dose group (HDG) were administered intragastrically by $50,100,200 \mathrm{mg} \cdot \mathrm{kg}^{-1}$ SCPs solutions (dissolved in $0.9 \%$ normal saline), respectively. During gavage, they drank water and ate the feed freely. The composition of basic diet is shown in Table S1. After treatment for 7, 14, or 21 days, six mice from each group were randomly chosen to collect the feces for measurement of water content and the colon tissues for further analysis. The colon tissues were weighed, measured the length, and then carefully scraped to collect the contents for high-throughput sequencing analysis after treatment for 21 days. Part of clean colon tissues was fixed in Bouin's fixative solution for histological observation. The remaining colon tissues in each group were cut, mixed, washed and shook with ultra-pure water, and then centrifuged at $2700 \times g$. The precipitation was collected in a sterile EP tube and frozen at $-80^{\circ} \mathrm{C}$ for determining the reducing sugar, monosaccharide, SCFAs contents.

\section{Histological observation of the colon}

After being fixed for $24 \mathrm{~h}$, the colon samples were dehydrated, cleared, paraffin embedded, sectioned, stained with hematoxylin and eosin (H\&E), and finally mounted. 
Afterwards the samples were observed via light microscopy (Olympus, Tokyo, Japan).

\section{Determination of fecal water content}

After being weighed $\left(\mathrm{m}_{2}\right)$, the feces were dried in an oven at $105 \pm 2{ }^{\circ} \mathrm{C}$ for several hours to constant weight, and then taken out to weigh $\left(\mathrm{m}_{3}\right)$ again.

Water content $(\%)=\left(\mathrm{m}_{2}-\mathrm{m}_{3}\right) / \mathrm{m}_{2} \times 100 \%$

\section{Detection of the reducing sugar, monosaccharide, and SCFAs contents of mice}

About $4 \mathrm{~g}$ colon tissues of mice in each group were cut into pieces, put in a $50 \mathrm{~mL}$ sterile centrifuge tube, washed repeatedly with ultra-pure water, and added the ultra-pure water to $50 \mathrm{~mL}$. After being centrifuged at $4000 \mathrm{r} / \mathrm{m}$, the supernatant was collected in the sterile EP tube for the determination of reducing sugar, monosaccharide, and SCFAs contents in colon contents by the DNS method [29, 30], gas chromatography [31], and solid-phase microextraction (SPME)-GC-MS (Thermo Fisher, Shanghai, China) as in vitro study.

\section{Preparation of total DNA and high-throughput sequencing analysis}

Fecal samples collected from the intestinal tissue of mice that had been fed for 21 days were used to extract DNA using the Soil DNA Kit (DNB100-50RXN; SigmaAldrich, St Louis, America). DNA was purified using 1\% agarose gel electrophoresis and then stored at $-80^{\circ} \mathrm{C}$.

High-throughput sequencing of the intestinal microbiota was carried out by the Baimike Biology Company, Beijing, China. Sequencing results were first spliced according to overlapping relationships, followed by quality control measurement, including filtering out sequence artifacts, and analysis for operational taxonomic unit (OTU) clustering of sequences, which was completed using Mothur software (https://www.mothur.org/) to assess the degree of species similarity between samples.

For the identification of differences in the functional genes among different intestine microbes, the Clusters of Orthologous Groups (COG) annotation was conducted against the COG database by BLASTp [32]. The Phylogenetic Investigation of Communities by Reconstruction of Unobserved States (PICRUSt) was used to predict the KEGG category [33].

\section{Statistical analysis}

Data are presented as the mean \pm standard deviation (SD). GraphPad Prism 5.0 software (GraphPad Software Inc., La Jolla, CA, America) was used for performing one-way ANOVA with a post hoc Tukey's test. $P<0.05$ indicated statistical significance.

\section{Results}

In vitro experiments

Effects of SCPs on biochemical indexes of fermentation broth

The results showed that the fermentation broth had an initial $\mathrm{pH}$ value of 6.53 and $\mathrm{OD}_{600}$ value of 0.21 . As fermentation progressed, the $\mathrm{pH}$ decreased gradually at first, reaching a low value of 5.17 after $24 \mathrm{~h}$ of incubation $(P<0.05)$, followed by an increase to 5.48 at the 48 -h time point $(P<0.05)$. Conversely, the $\mathrm{OD}_{600}$ increased gradually at first, and reached a maximum of 1.72 after $24 \mathrm{~h}$ of incubation $(P<0.05)$; afterwards the $\mathrm{OD}_{600}$ began to decrease, and the final value was 1.31 at the end of the experiment $(P<0.05)$ (Additional file 1: Figure S1).

Compared with the starting concentration, the total sugar content in the fermentation broth gradually decreased with the increase of fermentation time $(P<0.05)$. After $2 \mathrm{~h}$ of fermentation, the total sugar content in the broth decreased significantly $(P<0.05)$, and reached the lowest value at $24 \mathrm{~h}$. After that, the total sugar content was relatively unchangeable. However, the reducing sugar content in the fermentation broth firstly increased and then began to gradually decrease from 2 to $6 \mathrm{~h}$ of incubation, during which the concentration remained relatively stable until the experiment was terminated at $48 \mathrm{~h}$ (Table 1). Moreover, the content of reducing sugar was significantly increased compared with the starting concentration $(P<0.05)$.

Fermentation broth contained primarily five monosaccharides, namely, glucose, mannose, fructose, xylose, and galactose (Table 2). Throughout the fermentation process, the glucose content showed a downward trend $(P<0.05)$, while the concentrations of the other four monosaccharides all increased at first and then decreased, but their relative abundance differed at different time points. The glucose contents in the broth after fermentation from 1 to $48 \mathrm{~h}$ were remarkably reduced compared with the starting concentration $(P<0.05)$. Compared with the starting concentration, the mannose

Table 1 Effects of SCPs on the total sugar and reducing sugar content in the fermentation broth

\begin{tabular}{lll}
\hline Time $(\mathbf{h})$ & Total sugar $(\mathrm{mg} / \mathrm{mL})$ & Reducing sugar $(\mathrm{mg} / \mathbf{m L})$ \\
\hline 0 & $1.866 \pm 0.007^{\mathrm{a}}$ & $0.141 \pm 0.001^{\mathrm{a}}$ \\
1 & $1.762 \pm 0.004^{\mathrm{a}}$ & $0.677 \pm 0.001^{\mathrm{b}}$ \\
2 & $1.206 \pm 0.003^{\mathrm{b}}$ & $0.538 \pm 0.003^{\mathrm{b}}$ \\
6 & $0.933 \pm 0.001^{c}$ & $0.27 \pm 0.007^{c}$ \\
12 & $0.832 \pm 0.008^{c}$ & $0.211 \pm 0.003^{c}$ \\
24 & $0.703 \pm 0.007^{c}$ & $0.199 \pm 0.001^{c}$ \\
48 & $0.699 \pm 0.001^{c}$ & $0.153 \pm 0.009^{d}$ \\
\hline
\end{tabular}

Data represent mean $\pm \mathrm{SD}(n=3)$. a, b, c, and d represent significant differences among different time $(P<0.05)$ 
Table 2 Effects of SCPs on the monosaccharide components of the fermentation broth

\begin{tabular}{|c|c|c|c|c|c|}
\hline \multirow[t]{2}{*}{ Fermentation time (h) } & \multicolumn{5}{|c|}{ Relative amount (\%) } \\
\hline & Glucose & Mannose & Fructose & Xylose & Galactose \\
\hline 0 & $30.09 \pm 0.02^{\mathrm{a}}$ & $4.09 \pm 0.03^{\mathrm{a}}$ & $11.92 \pm 0.01^{\mathrm{a}}$ & $1.53 \pm 0.03^{\mathrm{a}}$ & $0.73 \pm 0.01^{\mathrm{a}}$ \\
\hline 1 & $21.23 \pm 0.03^{b}$ & $6.24 \pm 0.02^{b}$ & $19.77 \pm 0.01^{b}$ & $5.27 \pm 0.02^{b}$ & $1.74 \pm 0.04^{b}$ \\
\hline 2 & $9.33 \pm 0.07^{c}$ & $7.26 \pm 0.01^{b}$ & $20.34 \pm 0.02^{b}$ & $5.28 \pm 0.02^{b}$ & $2.03 \pm 0.03^{b}$ \\
\hline 6 & $3.06 \pm 0.01^{d}$ & $5.88 \pm 0.04^{c}$ & $10.77 \pm 0.05^{c}$ & $4.11 \pm 0.02^{b}$ & $2.87 \pm 0.01^{c}$ \\
\hline 12 & $1.29 \pm 0.01^{\mathrm{e}}$ & $3.54 \pm 0.01^{d}$ & $5.21 \pm 0.02^{d}$ & $3.02 \pm 0.01^{b}$ & $3.11 \pm 0.04^{c}$ \\
\hline 24 & - & $1.74 \pm 0.03^{e}$ & $3.11 \pm 0.03^{e}$ & $2.88 \pm 0.01^{b}$ & $2.03 \pm 0.03^{d}$ \\
\hline 48 & - & - & $1.89 \pm 0.02^{f}$ & $2.54 \pm 0.03^{b}$ & $0.43 \pm 0.01^{d}$ \\
\hline
\end{tabular}

Data represent mean $\pm \mathrm{SD}(n=3)$. a, b, c, and d represent significant differences among different time $(P<0.05)$

contents were obviously higher in the broth after fermentation from 1 to $6 \mathrm{~h}$, while were significantly lower in the broth after fermentation from 12 to $24 \mathrm{~h}(P<0.05)$. The fructose concentration after fermentation for $1-2 \mathrm{~h}$ was significantly higher than that at the starting time, while were remarkably lower from $6 \mathrm{~h}$ of fermentation $(P<0.05)$. The xylose contents after fermentation for $1-2 \mathrm{~h}$ were significantly increased $(P<0.05)$ and then decreased. During the whole fermentation process the xylose contents were obviously higher than the starting concentration. The galactose concentrations were significantly increased after fermentation for 1-12 $\mathrm{h}$ and then remarkably reduced. During the whole fermentation process, the galactose concentrations were significantly elevated compared with the starting concentration except $48 \mathrm{~h}(P<0.05)$.

As shown in Table 3, only certain SCFAs existed in the fermentation broth at the beginning of the fermentation process, namely, acetic, propionic, and butyric acid. As fermentation progressed, the relative total SCFAs contents increased significantly $(P<0.05)$. However, the highest concentration of specific SCFAs varied at different fermentation time. The contents of acetic acid, propionic acid, butyric acid reached the maximum after fermentation for $6,12,24 \mathrm{~h}$, respectively. The contents of pentanoic acid were detected after fermentation for $6 \mathrm{~h}$ and had the peak value for $48 \mathrm{~h}$. However, malonic acid and enanthic acid could not be detected until $48 \mathrm{~h}$ of fermentation.

\section{In vivo experiment \\ Effects of SPCs on fecal water content}

After treatment for 7, 14, 21 days with SCPs, the fecal water contents in all experimental mice were significantly higher than that in the control group $(P<0.01)$. During the whole experimental period, fecal water contents were increased firstly and then decreased in different SCP groups, while was decreased in the control group (Additional file 1: Figure. S2).

\section{Effects of SPCs on the colon physiology of mice}

The colonic index (Fig. 1a) in mice treated with SCPs was increased significantly compared with that in the control group, and colon length (Fig. 1b) was significantly increased in HDG group after treatment for 7 days and in all SCPs groups after treatment for 14 and 21 days compared with that in the control group $(P<0.01)$. Figure $1 \mathrm{c}$ shows that the epithelium

Table 3 Effects of SCPs on SCFAs in the fermentation broth

\begin{tabular}{lrrrrrrr}
\hline $\begin{array}{l}\text { Fermentation } \\
\text { time (h) }\end{array}$ & \multicolumn{2}{l}{ Relative amount (\%) } & & Total content (\%) \\
\cline { 2 - 6 } & \multicolumn{1}{l}{ Acetic acid } & Propionic acid & Butyric acid & Pentanoic acid & Malonic acid & Enanthic acid & \\
\hline 0 & $1.104 \pm 0.004^{\mathrm{a}}$ & $0.299 \pm 0.08^{\mathrm{a}}$ & $0.460 \pm 0.01^{\mathrm{a}}$ & - & - & - & $1.863 \pm 0.02^{\mathrm{a}}$ \\
1 & $3.105 \pm 0.007^{\mathrm{b}}$ & $2.001 \pm 0.02^{\mathrm{b}}$ & $1.817 \pm 0.03^{\mathrm{b}}$ & - & - & - & $6.923 \pm 0.04^{\mathrm{b}}$ \\
2 & $2.576 \pm 0.009^{\mathrm{b}}$ & $2.369 \pm 0.07^{\mathrm{b}}$ & $2.806 \pm 0.01^{\mathrm{b}}$ & - & - & - & $7.751 \pm 0.03^{\mathrm{b}}$ \\
6 & $18.354 \pm 0.08^{\mathrm{c}}$ & $2.806 \pm 0.09^{\mathrm{b}}$ & $6.5532 \pm 0.03^{\mathrm{c}}$ & $0.782 \pm 0.02^{\mathrm{a}}$ & - & - & $28.474 \pm 0.03^{\mathrm{c}}$ \\
12 & $3.381 \pm 0.03^{\mathrm{d}}$ & $39.698 \pm 0.06^{\mathrm{c}}$ & $20.884 \pm 0.02^{\mathrm{d}}$ & $0.782 \pm 0.03^{\mathrm{a}}$ & - & - & $64.745 \pm 0.06^{\mathrm{d}}$ \\
24 & $4.393 \pm 0.07^{\mathrm{d}}$ & $20.723 \pm 0.04^{\mathrm{d}}$ & $30.613 \pm 0.05^{\mathrm{e}}$ & $0.437 \pm 0.09^{\mathrm{b}}$ & - & - & $56.166 \pm 0.02^{\mathrm{e}}$ \\
48 & $13.478 \pm 0.03^{\mathrm{e}}$ & $3.45 \pm 0.03^{\mathrm{e}}$ & $9.177 \pm 0.08^{\mathrm{f}}$ & $35.489 \pm 0.07^{\mathrm{c}}$ & $0.070 \pm 0.04$ & $1.620 \pm 0.08$ & $65.481 \pm 0.11^{\mathrm{f}}$ \\
\hline
\end{tabular}

Data represent mean $\pm \mathrm{SD}(n=3)$. a, b, c, and d represent significant differences among different time $(P<0.05)$ 


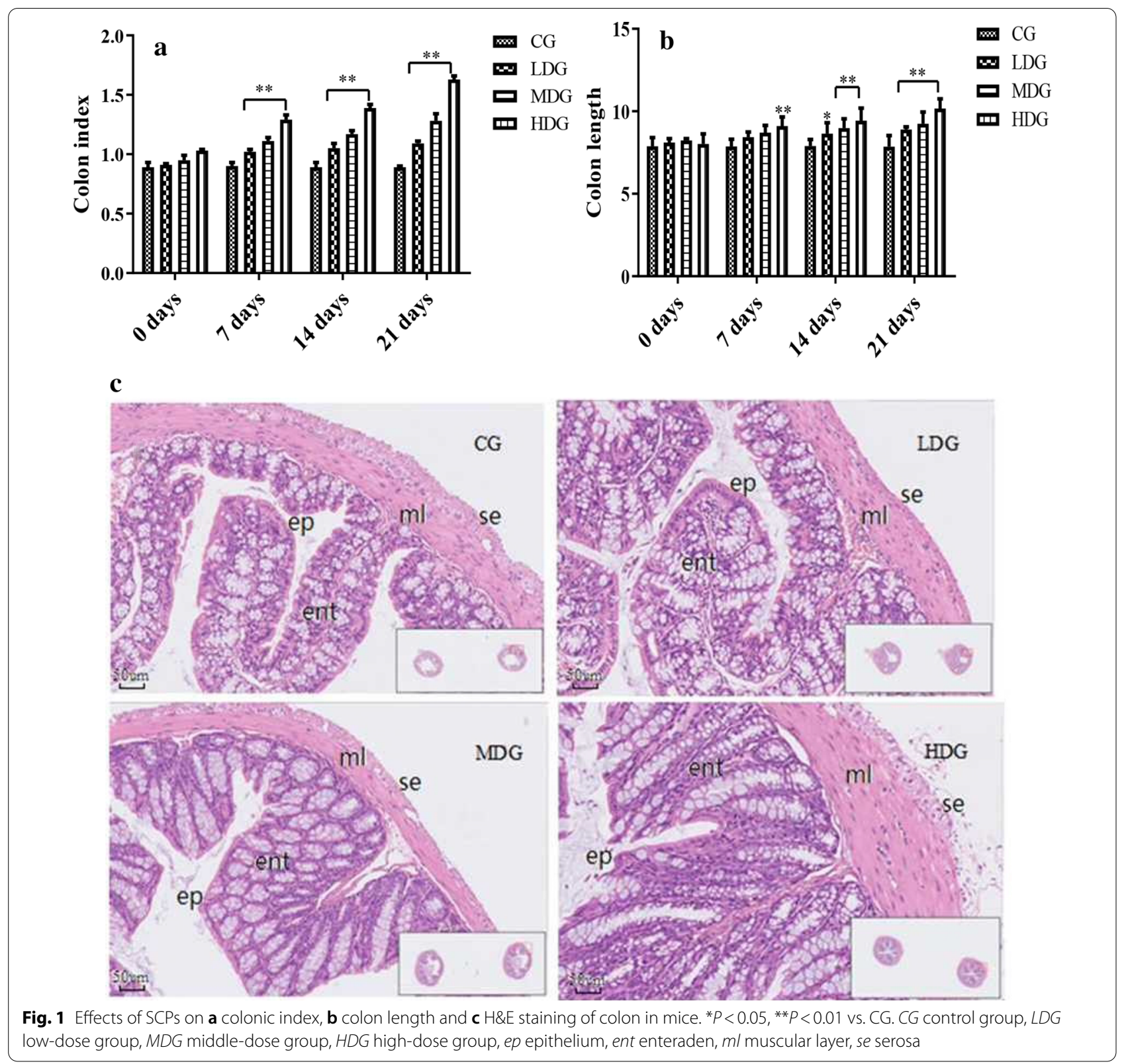

of colonic mucosa in the control group was complete with clear structure, orderly arranged epithelial cells, and the complete gland morphology. Compared with the control group, the volume of intestinal gland in the LDG group was increased, the lumen was narrowed slightly, and the serous membrane was thinned slightly. The muscle layer was thinned slightly, and the volume of intestinal gland was increased in MDG group. The lumen was narrowed and the muscle layer was thickened in HDG group. Moreover, the villi length of SCPstreated mice increased significantly compared with that of the control group $(P<0.05$, Fig. 1c).
Effects of SCPs on SCFAs, reduced sugar content and monosaccharides in intestinal contents of mice

Compared with the control group, total SCFAs contents in the intestinal contents of SCPs-treated mice were increased significantly $(P<0.01)$ in a dose-dependent manner (Fig. 2a). However, the relative abundance of each SCFAs varied during the course of the 21-day experiment. Acetic acid (Fig. 2b), butyric acid (Fig. 2d), and pentatonic acid (Fig. 2e) showed an upward trend during the experiment, while the relative abundance of propionic acid (Fig. 2c) began to decrease after 7 days, which may be due to its metabolic characteristics in vivo. 

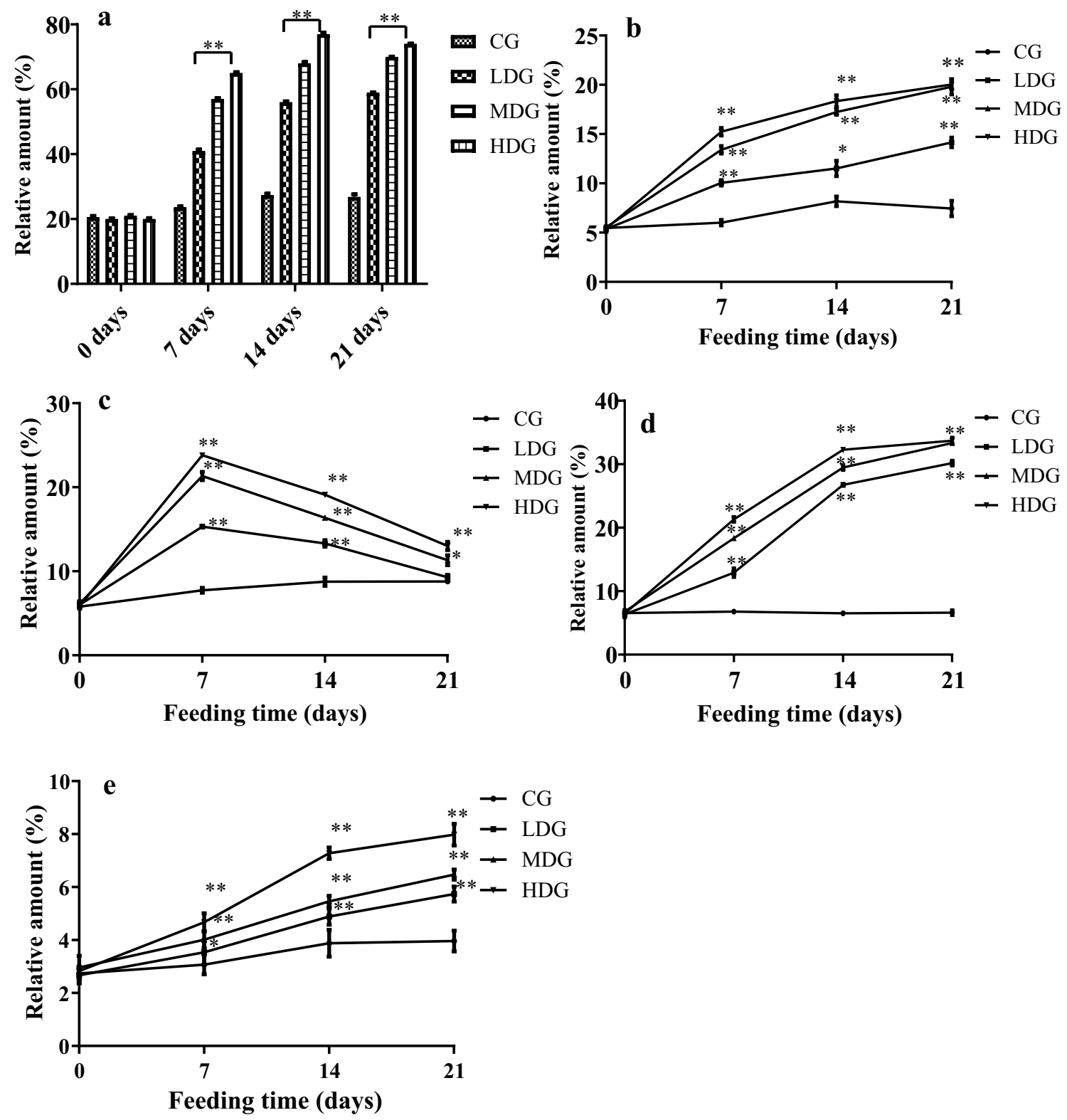

Fig. 2 Effects of SCPs on the relative abundance of SCFA in mouse intestinal content. ** $P<0.01$ vs. CG. CG control group, LDG low-dose group, MDG -dose group, HDG high-dose group. a Levels of total SCFAs, $\mathbf{b}$ levels of acetic acid, $\mathbf{c}$ levels of propionic acid, $\mathbf{d}$ levels of butyric acid, and $\mathbf{e}$ levels of pentanoic acid

The total reducing sugar content in the control group had a gradual decreasing trend, while that in all SCPstreatment groups increased within 14 days, followed by a slight decrease. Overall, compared with the control group, the total reducing sugar contents in the intestines of SCPs-treated mice were increased significantly $(P<0.05)$ (Additional file 1: Figure. S3).

The relative content of each monosaccharide in SCPs-treated mice was increased significantly
$(P<0.05, P<0.01)$ compared with that in the control group. Among them, the relative amounts of glucose (Fig. 3a), fructose (Fig. 3d), and galactose (Fig. 3e) in all SCPs groups demonstrated continuous upward trends. Meanwhile, the relative abundance of xylose and mannose (Fig. 3b, c) showed an initial upward trend followed by a slight decline after 14 and 21 days, respectively. 

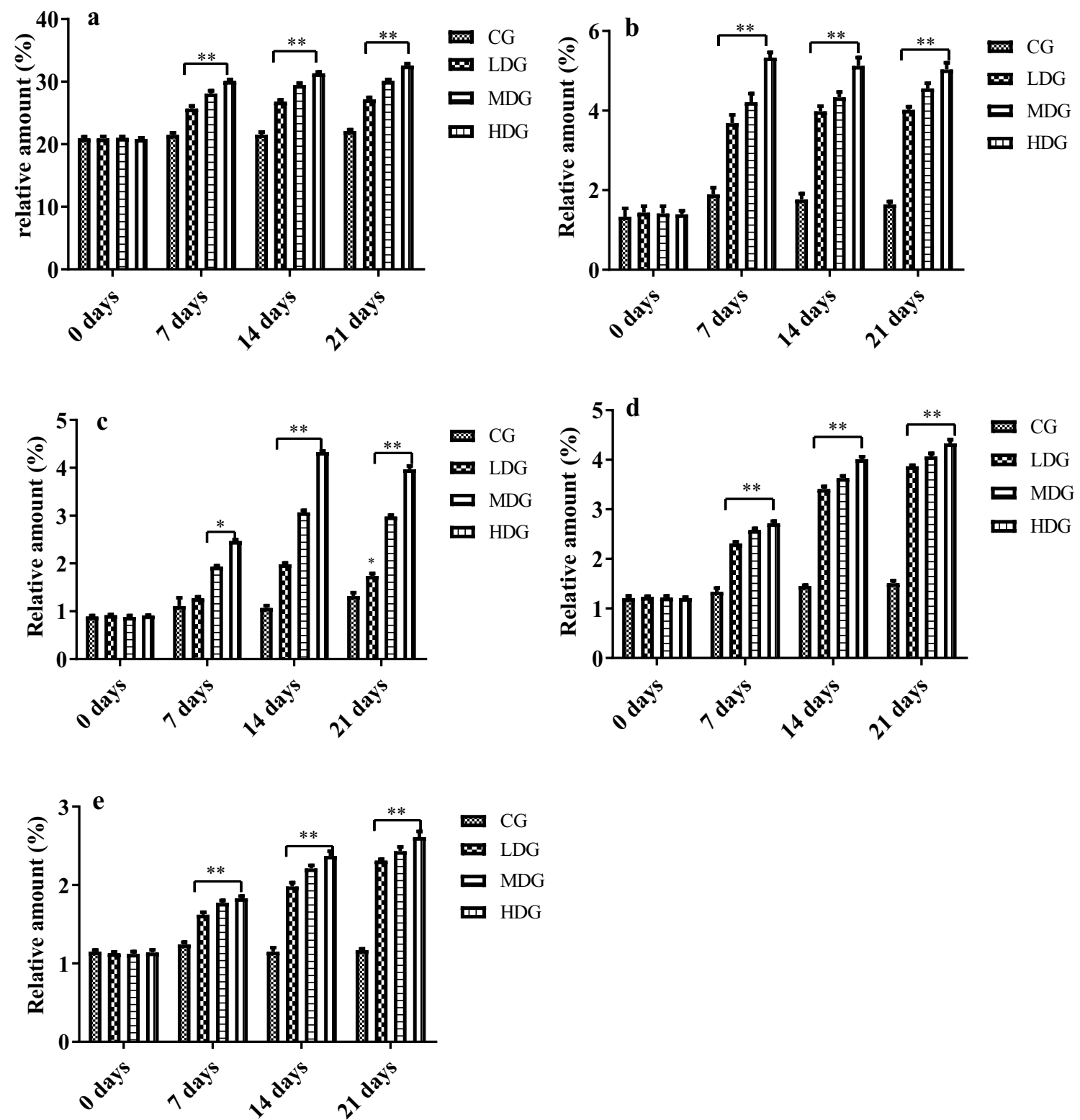

Fig. 3 Effects of SCPs on monosaccharide content in the intestinal content of mice. ${ }^{*} P<0.05 ;{ }^{* *} P<0.01 \mathrm{vs}$. CG. CG control group, LDG low-dose group, MDG middle-dose group, HDG high-dose group. a Levels of glucose, $\mathbf{b}$ levels of xylose, $\mathbf{c}$ levels of mannose, $\mathbf{d}$ levels of fructose, and $\mathbf{e}$ levels of galactose

\section{Effects of SCPs on intestinal microbiota in mice Effects of SCPs on the relative abundance of intestinal microbiota in mice at the phylum level}

The analysis of the phyla composition indicated that Bacteroidetes and Firmicutes were the most abundant intestinal microbiota in SCPs-treated mice and control mice, which accounted for approximately $97.11 \%$ of the total intestine microbes, followed by Proteobacteria, Cyanobacteria, and Deferribacteres (Fig. 4). Compared with the control group, the relative abundance of Firmicutes decreased with the increase of SCPs concentrations, and the percentage of Firmicutes in MDG and HDG was significantly lower by $11.02 \%$ and $16.01 \%$ than that in the CG $(P<0.01)$. Firmicutes had the lowest proportion in HDG, accounting for $45.66 \%$, which was significantly reduced compared with $54.37 \%$ in CG. The relative abundance of Bacteroidetes raised with the increase of SCPs concentrations, and the percentage of Bacteroidetes in MDG and HDG was significantly elevated by $12.51 \%$ and $17.57 \%$ compared with the CG $(P<0.01)$. The treatment 


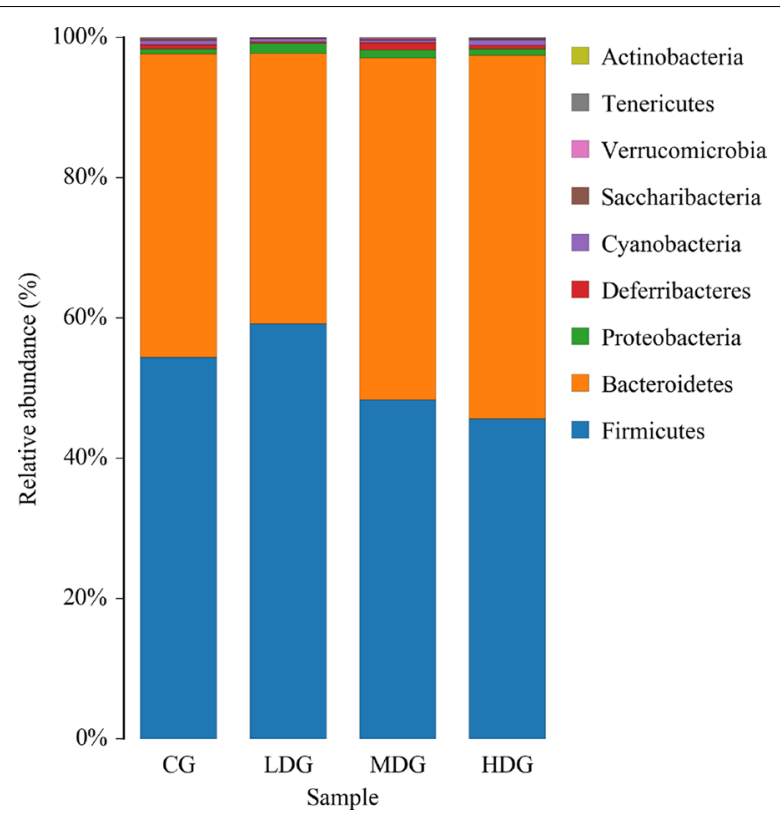

Fig. 4 Effects of SCPs on the relative abundance of intestinal flora in mice at the phylum level

with high-dose SCPs had the highest proportion of Bacteroidetes $(51.79 \%)$, which was remarkably higher than the CG $(43.31 \%)(P<0.01)$.

\section{Effects of SCPs on the relative abundance of intestinal microbiota in mice at the genus level}

At the genus level, compared with the control group, the relative abundance of Bacteroidales_S24-7_group, Alloprevotella, Bacteroides, Butyricimonas, and Parabacteroides in Bacteroidetes phylum increased with the increase of SCPs dose. The relative abundances of Bacteroidales_S24-7_group, Alloprevotella, Bacteroides, Butyricimonas, and Parabacteroides in the HDG were elevated by $22.18 \%, 35.61 \%, 63.86 \%, 94.30 \%$, and $295.24 \%$ compared with the CG, respectively (Fig. 5). While the relative abundance of Alistipes in Bacteroidetes phylum in the LDG was decreased by $5.31 \%$ compared to the CG. In Firmicutes phylum, the relative abundance of Oscillibacter and Lachnospiraceae_NK4A136_group in the HDG was increased by $93.75 \%$ and $22.03 \%$, while Ruminiclostridium was reduced with the increase of SCPs concentration, which were decreased by $24.24 \%$ in the HDG compared to the CG (Fig. 5).

\section{Functional prediction of gut microbiota induced by SCPs Analysis of metabolic pathways by KEGG}

KEGG analysis of metabolic pathways can reveal the differences and changes in microbial community functional genes in different metabolic pathways among different treatment groups. It can also show changes in metabolic function of intestinal microbiota in mice treated with different concentrations of SCPs. Treatment with SCPs resulted in differential expressions of 38 functional genes within the intestinal microbiota in three experimental groups $(P<0.05)$ in a non-dose-dependent manner. The results revealed that SCPs altered not only the composition and structure of intestinal microbiota in mice, but also the functional genes associated with metabolic pathways. This is likely due to the significant roles of intestinal microbiota in metabolism as well as in the endocrine, excretory, immune, circulatory, and digestive systems (Additional file 1: Figure. S4).

\section{Functional prediction and analysis of COG}

COG of proteins is a commonly used protein function classification database for prokaryotes. COG detects differences or changes in microbial functional proteins between different treatment groups. The results revealed that the functional proteins were detected in this experiment. Treatment with increasing concentrations of SCPs caused significant changes in the expressions of specific functional proteins within the intestinal microbiota of mice (Additional file 1: Figure. S5).

\section{Discussion}

We have shown here that polysaccharides are indeed fermented into monosaccharides by intestinal microbiota, which differs from the glycoside bonds that are broken down only in the digestive tract [34], and the glucose and mannose were the primary monosaccharides utilized by intestinal microorganisms during the fermentation of SCPs in vitro.

Due to the production of a large number of SCFAs and the proliferation of intestinal microbiota during the fermentation process, the change of $\mathrm{pH}$ value and $\mathrm{OD}_{600}$ value in the fermentation solution became important indicators in the fermentation process of SCPs. Since the intestinal microbiota in the human body can produce a certain amount of SCFAs, the initial $\mathrm{pH}$ and $\mathrm{OD}_{600}$ values in the fermentation broth were 6.53 and 0.21 , respectively. As fermentation progressed, more hydrogen ions were produced in the broth, thereby reducing the $\mathrm{pH}$ value. Thus, SCPs may have been fermented as a means to provide hydrogen ions to the environment. Meanwhile, the observed significant increase in $\mathrm{OD}_{600}$ might indicate that intestinal microorganisms could use polysaccharides to enhance proliferation and production of SCFAs, which was proved by the SCFAs content in the fermentation broth.

During the later stages of fermentation, the concentration of reducing sugars and total sugar decreased slowly, while the $\mathrm{pH}$ value increased. In addition, malonic acid 

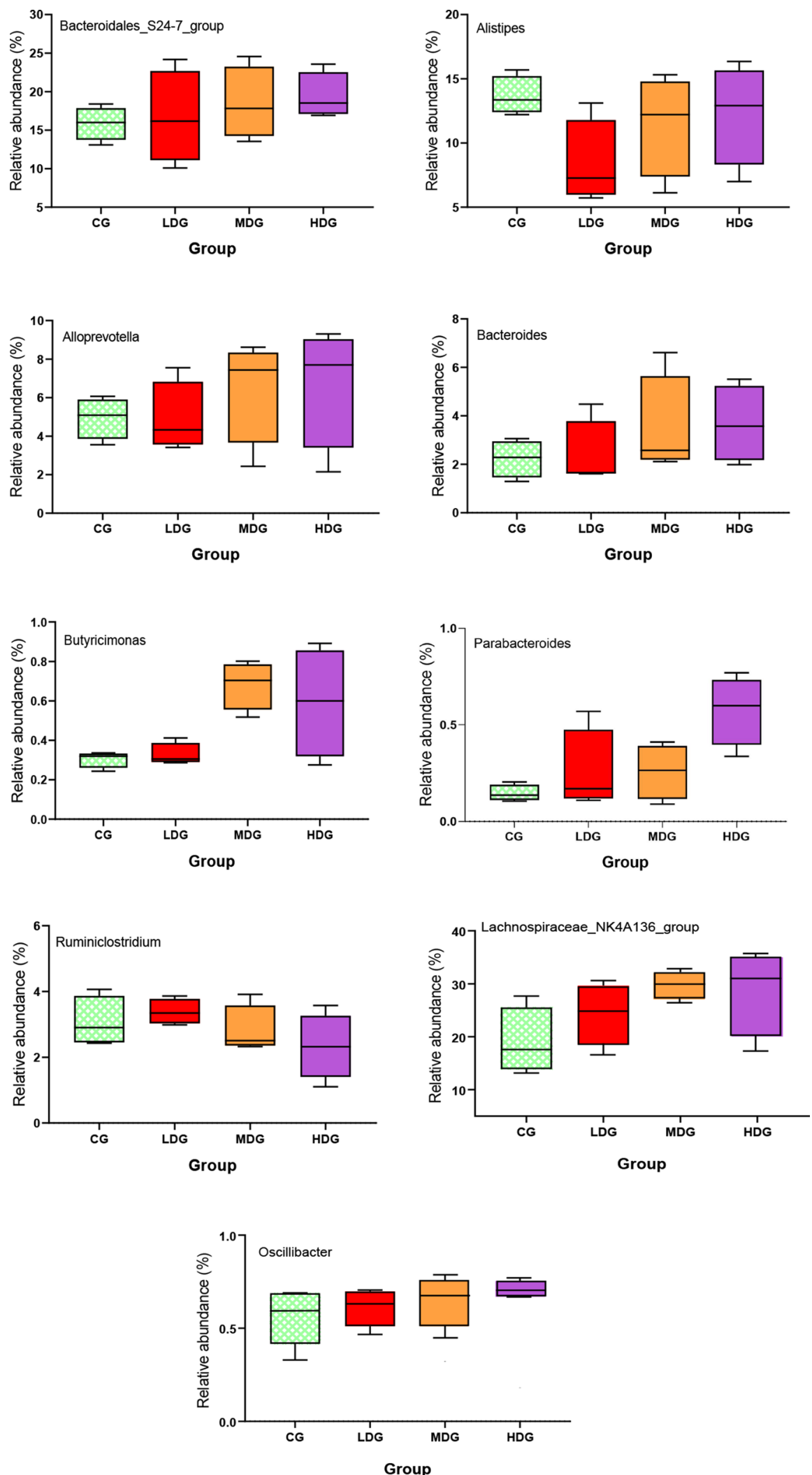

Fig. 5 Effects of SCPS on the relative abundance of intestinal flora in mice at the genus level 
and heptanoic acid were produced, which might indicate further fermentation of organic acids by intestinal microorganisms. Beneficial bacteria ferment polysaccharides to produce organic acids [35], the most common of which are butyric acid and propionic acid. These organic acids not only increase intestinal osmotic pressure, but also limit the process of putrefaction, which is beneficial to the human intestinal tract [36]. In this study, the total content of SCFAs in the fermentation broth increased significantly with the fermentation time, especially the propionic acid and butyric acid, which demonstrated that SCPs could gradually and continuously regulate the intestinal microenvironment to produce SCFAs. This result was proved by previous studies [15], which indicated that SCPs could be degraded by intestinal microbiota to produce SCFAs. It's worth noting that the major fermentation products were acetic acid, propionic acid, and butyric acid. The increased levels of SCFAs in vitro fermentation of SCPs might be associated with the release from the typical SCFAs-producing bacteria including Bacteroides, Bifidobacteria, Streptococcus and Butyricimonas [35]. It was interesting to note that the concentration of acetic acid in vitro fermentation of SCPs reached a maximum level at $6 \mathrm{~h}$, and then gradually dropped to a lower level at $24 \mathrm{~h}$, while the concentration of propionic acid and butyric acid increased gradually and reached a maximum level at 12 and $24 \mathrm{~h}$, respectively. It has been reported that about $24 \%$ of the colonic acetic acid is converted into butyric acid and $3 \%$ into propionic acids [37]. Therefore, we speculated that some of the acetic acid in vitro fermentation of SCPs might be converted into propionic acid and butyric acid during fermentation. The production of SCFAs can decrease the $\mathrm{pH}$ of the intestinal tract, promote gastrointestinal motility, and inhibit the growth and proliferation of pathogenic bacteria and harmful bacteria including $E$. coli, thus prevent the occurrence of intestinal diseases and tumors $[38,39]$. Similar to in vitro results, SCFAs in the intestinal content of our mouse model also increased significantly after treatment with SCPs compared with control group. Moreover, the relative concentration of butyric acid and acetic acid in SCP-treated groups was shown to increase significantly. However, the concentration of propionic acid was observed to firstly increase and then decrease during the experiment, which might be due to a physiological requirement, wherein only a small amount of propionic acid must remain in the intestinal tract, while the rest is transferred to the circulatory system. Moreover, propionic acid and acetic acid produced by intestinal microbiota metabolism are carried to the liver for de novo synthesis of even-carbon fatty acids and SCFAs, respectively [40]. It has also been reported that acetic acid and propionic acid have anti-inflammatory effects, both acetic and butyric acid can regulate the composition and structure of intestinal microbiota, and butyric acid is an important energy source for the intestinal mucosa [41]. Therefore, the increase in specific SCFAs in our in vivo study may have a beneficial role in maintaining intestinal microbes. Additionally, it is reported that butyric acid can promote postprandial insulin secretion, which may be one of the mechanisms of polysaccharides reducing blood sugar [42]. Thus, SCPs have the potential to reduce blood sugar by regulating the content of SCFAs. The specific molecular mechanism requires further investigation.

The microbiota in the gut can ferment the most extraneous polysaccharides, stimulate the growth and/ or the activity of some communities, and thus have an effect on the host's health [18]. In this study, Bacteroidetes and Firmicutes were the most abundant microbial phyla in the intestines of SCPs-treated mice and control mice. Treatment with SCPs couldn't change the dominant intestinal microbiota of mice at the phylum level. Bacteroidetes and Firmicutes account for $>90 \%$ of the bacterial population in the colon [43]. Low diversity of intestinal microbiota at phyla level may be caused by the host's selective pressure on the composition and function of gastrointestinal microbiome and the relatively short human evolutionary history compared with the long history of the Earth [44]. However, treatment with SCPs could significantly increase the relative abundance of Bacteroidetes spp. in the intestinal tract while simultaneously decrease the relative abundance of Firmicutes spp., which indicated that SCPs had certain regulatory effects on the structure of intestinal microbiota in mice. The Similar results were obtained in the study by Ren et al., which found that seleno-lentinan could improve the relative abundance of Bacteroidetes and reduce the relative abundance of Firmicutes [45]. Previous studies showed that the decrease in the ratio of Bacteroides to Firmicutes (B:F) has been associated with many disease conditions [46]. Feeding polysaccharide can reverse the ratio of Bacteroides and Firmicutes [47, 48]. Treatment with SCPs could significantly increase the Bacteroidetes/Firmicutes ratio in the intestinal tract, which indicated that SCPs could regulate the microbiota structure in the intestinal tract of mice. The increase of the Bacteroidetes/Firmicutes ratio might be caused by $\beta-(1 \rightarrow 3)$-D-glucan, the major structural units of SCPs, which has been found to stimulate and increase the levels of probiotics in the intestine [49]. Similar shifts in Bacteroidetes to Firmicutes ratio were observed in paramylon-treated mice who received high molecular weight $\beta$-glucan derived from microalgae [19]. Further, in silico predictions found that the functions of microbiota related to glycan utilization and glycerolipid metabolism were enhanced 
after treated with the $\beta$-glucans [19]. The increased ratio of Bacteroides and Firmicutes exerted a net positive effect on intestinal health.

Many beneficial intestinal bacteria belong to the genus Bacteroides, and their main function is to degrade plant polysaccharides that are difficult for the human body to breakdown [50]. Thus, the intestinal microbiota could strengthen the intestinal mucosa barrier, prevent intestinal dysfunction, and inhibit colonic inflammation [51]. In addition to the shift at the phylum level, the SCPs changed some important communities in antitumor-related genus level. The relative abundance of Bacteroidales_S24-7_group, Alloprevotella, Alistipes, Bacteroides, Butyricimonas, and Parabacteroides in Bacteroidetes phylum in the intestinal tract of SCPs-treated mice were increased, while the relative abundance of Lachnospiraceae_NK4A136 group and Oscillibacter in Firmicutes phylum were decreased. It has been reported that the fermentation of polysaccharides in the gut produced SCFAs and some other prebiotics by gut bacteria to supply the nutrients for bacterial metabolism [52]. For example, the relative abundance of Bacteroides spp. was increased by feeding SCPs, it is possible that the amount of SCFAs may increase in this case. The Bacteroidales_S24-7_group was considered to be the main member of the intestinal microbiota and was recognized as a new branch of $\mathrm{Bac}$ teroides in 2002 [53]. The increased relative abundance of Bacteroidales_S24-7_group genus could relieve the colitis in mice feeding high-fat diet [54], which was consistent with our result. Butyricimonas has been found to be capable of producing SCFAs [55]. Parabacteroides is thought to be associated with the synthesis of SCFAs, the increase of which enhances its protective effects on the intestinal mucosal barrier and decreases the levels of enterogenous endotoxins, proved by the increase of SCFAs and the improved physiological state of the colon [56]. Alistipes was further enriched after feeding with SCPs. Previous studies showed that Alistipes, a major SCFAs producer, could reduce intestinal inflammation and played an important role in suppression of tumor growth [57, 58]. The Lachnospiraceae is known to participate in the carbohydrate fermentation into SCFAs and gases $\left(\mathrm{CO}_{2}\right.$ and $\left.\mathrm{H}_{2}\right)$ in the human intestine [59]. The increase of SCFAs will lead to the decrease of colonic $\mathrm{pH}$, ammonia production and absorption in the gut, therefore, Lachnospiraceae is good for the intestinal health [38]. The increase of Lachnospiraceae in SCPs-treated mice was consistent with these findings. Oscillibacter is a valerate producer and capable of enhancing the differentiation of IL-10 producing Tregs in vivo [60], which was proved by the valerate content in the gut of mice. However, the relative abundance of Ruminiclostridium was reduced significantly. The Ruminiclostridium belongs to Ruminococcaceae, which was closely associated with depression [51].

The colon functions to absorb water and salt from food before it is discharged from the body and has an integral role in maintaining intestinal microbes. Intestinal diseases can lead to a decreased colonic index, colon length. Moreover, the growth state of the colon can determine the efficiency of polysaccharide hydrolysis [61]. The intake of carbohydrates increases the wet weight of feces resulting in a more relaxed colonic state, which is considered to have a positive impact on colon health [62]. In this study, the protective effect of SCPs on colonic tissue was observed, which was characterized by significantly increase of the colonic index, the length of colon and villi, and the fecal water content in SCP-treated mice compared with those of the control group, suggesting that the intake of SCPs might improve the physiological state of the colon. This colonic mucosa prevention may be associated with colon bacterial fermentation of SCPs and the intestinal function regulation of their metabolites of SCFAs, which can not only be used as energy source for colonocytes to inhibit the growth of harmful pathogens, but also be used as an in vivo lipogenesis of the intestinal microbiota or the liver [63].

The metabolites of intestinal microbiota play an important role in intestinal microbiota ecology and host-microbiome interactions [64]. In agreement with the structural changes in the gut microbiota, SCPs treatment significantly influenced the predictive functions and intestinal microbiota metabolism, evidenced by significant changes of functions related with carbohydrate metabolism, amino acid metabolism, energy metabolism. This is in accordance with the utilization of SCPs and subsequent SCFAs production [65], proved by previous studies which showed that long transit times of carbohydrate in the large intestine had profound effects on the physiology and metabolism of bacteria, leading to amino acid fermentation, thereby making an increased contribution to colon SCFAs pools [66]. Most of the overrepresented predicted functions observed in the gut microbiota of control mice were linked to Firmicutes, primarily to the genus Oscillibacter. Conversely, most of the overrepresented metabolic pathways observed in the gut microbiota of SCPs-treated mice were related to Bacteroides, which has many species capable of SCFAs synthesis and modulation of the immune system [67]. The specific mechanism of SCPs regulating the carbohydrate metabolism, amino acid metabolism, energy metabolism through gut microflora needs further investigation. 


\section{Conclusion}

In summary, this study demonstrates that during the intestinal microbiota fermentation processes, SCPs were utilized by intestinal microbiota to produce SCFAs. Moreover, SCPs can improve the physiological indices of the colon in mice by increasing the relative abundance and diversity of SCFA-producing bacteria and SCFAs level produced by intestinal microbiota. Via KEGG and COG analysis of the intestinal microbiota, the changes in the intestinal microbiota after treatment with SCPs altered specific functional proteins and metabolic pathways that might reduce the occurrence of various diseases. SCPs can increase the relative abundance of dominant intestinal bacteria, thus optimize the composition of the intestinal microbiota. Our study showed the promising effects of SCPs on maintaining healthy gut microbes, which may contribute to exert profound health promoting effects.

\section{Supplementary Information}

The online version contains supplementary material available at https://doi. org/10.1186/s40538-021-00225-8.

Additional file 1. Additional figures.

\section{Acknowledgements}

This project was supported by the Key Research Plan Projects of Shanxi Province (201603D211201); the Collaborative Innovation Center for Enhancing Quality and Efficiency of Loess Plateau Edible Fungi; Scientific and Technological Innovation Team of Edible Mushroom in Shanxi Province; Special Scientific Research Project of Agricultural Valley Construction in Shanxi Province (SXNGJSKYZX201903).

\section{Authors' contributions}

CF contributed to the conception of the study; $M-Y L, M-C C, J-L M, J-Y L$, Y-FC, L-JX, X-RG performed the experiment; CF, S-JY, J-LC, FC contributed significantly to analysis and manuscript preparation; M-YL performed the data analyses and wrote the manuscript; S-JY, J-LC helped perform the analysis with constructive discussions. All authors read and approved the final manuscript.

\section{Funding}

This project was supported by the Key Research Plan Projects of Shanxi Province (201603D211201); the Collaborative Innovation Center for Enhancing Quality and Efficiency of Loess Plateau Edible Fungi; Special Scientific Research Project of Agricultural Valley Construction in Shanxi Province (SXNGJSKYZX201903).

\section{Availability of data and materials}

The datasets used or analyzed during the current study are available from the corresponding author on reasonable request.

\section{Declarations}

\section{Ethical approval and consent to participate}

All animal treatments were performed in accordance with the Provisions and General Recommendation of Chinese Experimental Animals Administration Legislation.

\section{Consent for publication}

Written informed consent for publication was obtained from all participants.

\section{Competing interests}

The authors declare no conflicts of interest.

\section{Author details}

${ }^{1}$ College of Food Science and Engineering, Shanxi Agricultural University, Taigu, Shanxi 030801, The People's Republic of China. ${ }^{2}$ Shanxi Research Station for Engineering Technology of Edible Fungi, Taigu, Shanxi 030801, The People's Republic of China.

Received: 6 March 2021 Accepted: 22 April 2021

Published online: 19 May 2021

\section{References}

1. Kho ZY, Lal SK. The human gut microbiome-a potential controller of wellness and disease. Front Microbiol. 2018;9:1835.

2. Illiano P, Brambilla R, Parolini C. The mutual interplay of gut microbiota, diet and human disease. FEBS J. 2020;287(5):833-55.

3. Li S, Qi Y, Chen L, Qu D, Li Z, Gao K, Chen J, Sun Y. Effects of Panax ginseng polysaccharides on the gut microbiota in mice with antibiotic-associated diarrhea. Int J Bio Macromol. 2019;124:931-7.

4. Gowd V, Bao T, Wang L, Huang Y, Chen S, Zheng X, Cui SL, Chen W. Antioxidant and antidiabetic activity of blackberry after gastrointestinal digestion and human gut microbiota fermentation. Food Chem. 2018;269:618-27.

5. Zorraquín I, Sánchez-Hernández E, Ayuda-Durán B, Silva M, GonzálezParamás AM, Santos-Buelga C, Moreno-Arribas MV, Bartolome B. Current and future experimental approaches in the study of grape and wine polyphenols interacting gut microbiota. J Sci Food Agric. 2020;100:3789-802.

6. Yu HC, Qiu N, Meng YQ, Keast R. A comparative study of the modulation of the gut microbiota in rats by dietary intervention with different sources of egg-white proteins. J Sci Food Agric. 2020;100:3622-9.

7. Chen $W$, Su HM, Xu Y, Jin C. In vitro gastrointestinal digestion promotes the protective effect of blackberry extract against acrylamide-induced oxidative stress. Sci Rep. 2017;7:41514.

8. Su HM, Xie LH, Xu Y, Ke HH, Bao T, Li YT, Chen W. Pelargonidin-3-Oglucoside derived from wild raspberry exerts antihyperglycemic effect by inducing autophagy and modulating gut microbiota. J Agric Food Chem. 2020;68:13025-37

9. Broom LJ, Kogut MH. Gut immunity: its development and reasons and opportunities for modulation in monogastric production animals. Anim Health Res Rev. 2018;19:46-52.

10. Yu Z, Song G, Liu J, Wang J, Zhang P, Chen K. Beneficial effects of extracellular polysaccharide from Rhizopus nigricans on the intestinal immunity of colorectal cancer mice. Int J Bio Macromol. 2018;115:718-26.

11. Sun SS, Wang K, Sun L, Cheng BS, Qiao SS, Dai HQ, Shi WY, Ma JC, Liu $\mathrm{HM}$. Therapeutic manipulation of gut microbiota by polysaccharides of Wolfiporia cocos reveals the contribution of the gut fungi-induced $\mathrm{PGE}_{2}$ to alcoholic hepatic steatosis. Gut Microbes. 2020;12(1):e1830693.

12. Yu S, Jiang J, Li W. Co-cultured Lepista sordida and Pholiota nameko polysaccharide-iron (iii) chelates exhibit good antioxidant activity. RSC Adv. 2020;10(46):27259-65.

13. Ke H, Bao T, Chen W. Polysaccharide from Rubus chingii Hu affords protection against palmitic acid-induced lipotoxicity in human hepatocytes. Int J Biol Macromol. 2019;133:1063-71.

14. Lee YE, Yoo SH, Chung JO, Park MY, Hong YD, Park SH, Park TS, Shim SM. Hypoglycemic effect of soluble polysaccharide and catechins from green tea on inhibiting intestinal transport of glucose. J Sci Food Agric. 2020;100:3979-86.

15. Liu YT, Li YW, Ke Y, Li C, Zhang ZQ, Hu B, Liu AP, Luo QY, Wu WJ. In vitro saliva-gastrointestinal digestion and fecal fermentation ofOudemansiella radicata polysaccharides reveal its digestion profile andeffect on the modulation of the gut microbiota. Carbohyd Polym. 2021;251:11704.

16. Huang F, Hong R, Zhang R, Yi Y, Dong L, Liu L, Jia X, Ma Y, Zhang M. Physicochemical and biological properties of longan pulp polysaccharides modified by Lactobacillus fermentum fermentation. Int J Bio Macromol. 2019;125:232-7.

17. Huang X, Nie S, Xie M. Interaction between gut immunity and polysaccharides. Crit Rev Food Nutr. 2017:57(14):2943-55. 
18. Xu X, Xu P, Ma C, Tang J, Zhang X. Gut microbiota, host health, and polysaccharides. Biotechnol Adv. 2013;31:318-37.

19. Chen L, Liu J, Ge X, Xu W, Chen Y, Li F, Cheng D, Shao R. Simulated digestion and fermentation in vitro by human gut microbiota of polysaccharides from Helicteres angustifolia L. Int J Biol Macromol. 2019;141:1065-71.

20. Xu X, Zhang X. Lentinula edodes-derived polysaccharide alters the spatial structure of gut microbiota in mice. PLoS ONE. 2015;10(1):e0115037.

21. Fu Y, Zhang J, Chen K, Xiao C, Fang B. An in vitro fermentation study on the effects of Dendrobium officinale polysaccharides on human intestinal microbiota from fecal microbiota transplantation donors. J Funct Foods. 2019:53:44-53.

22. Han JM, Lee EK, Gong SY, Sohng JK, Jung HJ. Sparassis crispa exerts anti-inflammatory activity via suppression of TLR-mediated NF-KB and MAPK signaling pathways in LPS-induced RAW264.7 macrophage cells. Ethnopharmacol. 2019;231:10-8.

23. Song WR, Yan J, Wu F, Bao HY. A study on antitumor effect of ultramicro powder of Sparassis latifolia. Mycosystema. 2017;36(12):1651-8.

24. Hida TH, Kawaminami H, Ishibashi K, Miura NN, Adachi Y, Ohno N. Ora administration of soluble $\beta$-glucan preparation from the cauliflower mushroom, Sparassis crispa (higher basidiomycetes) modulated cytokine production in mice. Int J Med Mushrooms. 2013;15(6):525-38.

25. Kim HH, Lee S, Singh TS, Choi JK, Shin TY, Kim SH. Sparassis crispa suppresses mast cell-mediated allergic inflammation: role of calcium, mitogen-activated protein kinase and nuclear factor-KB. Int J Mol Med. 2012;30:344-50

26. Hu S, Wang D, Zhang JR, Du MY, Cheng YK, Liu Y, Zhang N, Wang D, Wu Y. Mitochondria related pathway is essential for polysaccharides purified from Sparassis crispa mediated neuro-protection against glutamateinduced toxicity in differentiated pc12 cells. Int J Mol Sci. 2016:17(2):133.

27. Bulam S, Üstün NŞ, Pekşen A. $\beta$-glucans: An important bioactive molecule of edible and medicinal mushrooms, ed. By Türkmen A. 2018;1:1242-58.

28. Hao ZQ, Chen ZJ, Chang MC, Meng JL, Liu JY, Feng CP. Rheological properties and gel characteristics of polysaccharides from fruit-bodies of Sparassis crispa. Int J Food Prop. 2018;21:2283-95.

29. Nielsen SS. Phenol-sulfuric acid method for total carbohydrates. In: Nielsen SS, editor. Food analysis laboratory manual. Food science texts series. Boston: Springer; 2010. p. 47-53.

30. Wang JG, Zhang SZ, Yang BP, Cheng P, Wu ZD, Hu JY. Application of 3,5-dinitrosalicylic acid (dns) method to test the reducing sugar and water-soluble total sugar content in sugarcane internodes. Sugarcane Canesugar. 2008;5:45-9.

31. Wang X, Zhang L, Wu J, Xu W, Lü X. Improvement of simultaneous determination of neutral monosaccharides and uronic acids by gas chromatography. Food Chem. 2017;220:198-207.

32. Tatusov RL, Galperin MY, Natale DA, Koonin EV. The COG database: a tool for genome-scale analysis of protein functions and evolution. Nucleic Acids Res. 2000;28:33-6.

33. Langille MGI, Zaneveld J, Caporaso JG, McDonald D, Knights D, Reyes JA, Clemente JC, Burkepile DE, Thurber RLV, Knight R, Beiko RG, Huttenhower C. Predictive functional profiling of microbial communities using $16 \mathrm{~S}$ rRNA marker gene sequences. Nat Biotechnol. 2013:31:814-21.

34. Song EH, Shang J, Ratner DM. Polysaccharides. Poly Sci. 2012;9:137-55.

35. Wu Y, Chen Y, Lu Y, Hao H, Liu J, Huang R. Structural features, interaction with the gut microbiota and anti-tumor activity of oligosaccharides. RSC Adv. 2020;10:16339-48.

36. Zhou M, Pu C, Xia L, Yu X, Zhu B, Cheng R, Xu L, Zhang J. Salecan diet increases short chain fatty acids and enriches beneficial microbiota in the mouse cecum. Carbohyd Polym. 2014;102:772-9.

37. Huang J, Wang $Q, X u Q$, Zhang $Y$, Zheng Y. In vitro fermentation of O-acetyl-arabinoxylan from bamboo shavings by human colonic microbiota. Int J Biol Macromol. 2019;125:27-34.

38. Shen $\mathrm{H}, \mathrm{Xu} Z$ Z, Shen Z, Lu Z. The regulation of ruminal short-chain fatty acids on the functions of rumen barriers. Front Physiol. 2019;10:1305.

39. Pan J, Yin J, Zhang K, Xie P, Ding H, Huang X, Blachier F, Kong X. Dietary xylo-oligosaccharide supplementation alters gut microbial composition and activity in pigs according to age and dose. AMB Express. 2019;9(1):134

40. Zhai X, Lin D, Zhao Y, Li W, Yang X. Effects of dietary fiber supplementation on fatty acid metabolism and intestinal microbiota diversity in c57bl/6j mice fed with a high-fat diet. J Agric Food Chem. 2018;66(48):12706-18.
41. Molina S, Moran-Valero MI, Martin D, Vázquez L, Reglero G. Anti-proliferative effect of alkylglycerols as vehicles of butyric acid on colon cancer cells. Chem Phys Lipids. 2013;175-176:50-6.

42. Sanna S, van Zuydam NR, Mahajan A, Kurilshikov A, Vich Vila A, Võsa U, Mujagic Z, Masclee AAM, Jonkers DMAE, Oosting M, Joosten LAB, Netea MG, Franke L, Zhernakova A, Fu J, Wijmenga C, McCarthy MI. Causal relationships among the gut microbiome, short-chain fatty acids and metabolic diseases. Nat Genet. 2019;51:600-5.

43. Ley RE, Hamady M, Lozupone C, Turnbaugh PJ, Ramey RR, Bircher JS, Schlegel ML, Tucker TA, Schrenzel MD, Knight R. Evolution of mammals and their gut microbes. Science. 2008;320:1647-51.

44. Youngblut ND, Reischer GH, Walters W, Schuster N, Walzer C, Stalder G, Ley RE, Farnleitner AH. Host diet and evolutionary history explain different aspects of gut microbiome diversity among vertebrate clades. Nat Commun. 2019;10:2200.

45. Ren GM, Yu M, Li KK, Hu Y, Wang Y, Xu XH, Qu JJ. Seleno-lentinan prevents chronic pancreatitis development and modulates gut microbiota in mice. $J$ of Funct Foods. 2016;22:177-88.

46. Bolam DN, Sonnenburg JL. Mechanistic insight into polysaccharide use within the intestinal microbiota. Gut Microbes. 2011:2:86-90.

47. Brown K, DeCoffe D, Molcan E, Gibson DL. Diet-induced dysbiosis of the intestinal microbiota and the effects on immunity and disease. Nutrients. 2012:4:1095-119.

48. Jin M, Zhu Y, Shao D, Zhao K, Xu C, Li Q. Effects of polysaccharide from mycelia of Ganoderma lucidum on intestinal barrier functions of rats. Int Bio Macromol. 2017;94:1-9.

49. Jayachandran M, Chen J, Chung SSM, Xu B. A critical review on the impacts of $\beta$-glucans on gut microbiota and human health. J Nutr Biochem. 2018;61:101-10.

50. Li K, Zhuo C, Teng C, Yu S, Wang X, Hu Y. Effects of Ganoderma lucidum polysaccharides on chronic pancreatitis and intestinal microbiota in mice. Int J Bio Macromol. 2016;93:904-12.

51. Jädert C, Phillipson M, Holm L, Lundberg JO, Borniquel S. Preventive and therapeutic effects of nitrite supplementation in experimental inflammatory bowel disease. Redox Bio. 2014;2:73-81.

52. Neyrinck AM, Possemiers S, Druart C, Van de Wiele T, De Backer F, Cani PD, Larondelle Y, Delzenne NM. Prebiotic effects of wheat arabinoxylan related to the increase in bifidobacteria, Roseburia And Bacteroides/ Prevotella in diet-induced obese mice. PLoS ONE. 2011;6:e20944.

53. Ormerod KL, Wood DLA, Lachner N, Gellatly SL, Daly JN, Parsons JD. Genomic characterization of the uncultured Bacteroidales family S24-7 inhabiting the guts of homeothermic animals. Microbiome. 2016:4:36.

54. Rooks MG, Garrett WS. Gut microbiota, metabolites and host immunity. Nat Rev Immunol. 2016:16:341-52.

55. Bui TPN, Ritari J, Boeren S, De Waard P, Plugge CM, De Vos WM. Production of butyrate from lysine and the Amadori product fructoselysine by a human gut commensal. Nat Commun. 2015;6:10062.

56. Shen F, Zheng RD, Sun XQ, Ding WJ, Wang XY, Fan JG. Gut microbiota dysbiosis in patients with non-alcoholic fatty liver disease. Hepatobiliary Pancreat Dis Int. 2017;16:375-81.

57. lida N, Dzutsev A, Stewart CA, Smith L, Bouladoux N, Weingarten RA, et al. Commensal bacteria control cancer response to therapy by modulating the tumor microenvironment. Science. 2013:342:967-70.

58. Dziarski R, Park SY, Kashyap DR, Dowd SE, Gupta D. Phlyrp-regulated gut microflora Prevetella falsenii, Alistipes finegoldii attenuated colitis in mice. PLOS ONE. 2016;11:e0146162.

59. Duncan $\mathrm{SH}$, Louis P, Flint HJ. Cultivable bacterial diversity from the human colon. Lett Appl Microbiol. 2007:44:343-50.

60. Arpaia N, Campbell C, Fan XY, Dikiy S, van der Veeken J, deRoos P, Liu H, Cross JR, Pfeffer K, Coffer PJ, Rudensky AY. Metabolites produced by commensal bacteria promote peripheral regulatory T-cell generation. Nature. 2013:504:451-5.

61. Mudd AT, Berding K, Wang M. Serum cortisol mediates the relationship between fecal Ruminococcus \& brain N-acetylaspartate in the young pig. Gut Microbes. 2017;8:589-600.

62. Amsterdam A. [Retraction] Use of multiple biomarkers for the localization and characterization of colon cancer stem cells by indirect immunocytochemistry. Int J Oncol. 2012;41(5):1888.

63. Arcila JA, Weier SA, Rose DJ. Changes in dietary fiber fractions and gut microbial fermentation properties of wheat bran after extrusion and bread making. Food Res Int. 2015;74:217-23. 
64. Tramontano M, Andrejev S, Pruteanu M, Klünemann M, Kuhn M, Galardini M, Jouhten P, Zelezniak A, Zeller G, Bork P, Typas A, Patil K. Nutritional preferences of human gut bacteria reveal their metabolic idiosyncrasies. Nat Microbiol. 2018;3:514-22.

65. Miyamoto J, Watanabe K, Taira S, Kasubuchi M, Li Xuan Irie J, Itoh H, Kimura I. Barley $\beta$-glucan improves metabolic condition via short-chain fatty acids produced by gut microbial fermentation in high fat diet fed mice. PLoS ONE. 2018;13:e0196579.

66. Macfarlane S, Macfarlane GT. Regulation of short-chain fatty acid production. P Nutr Soc. 2003;62(1):67-72.
67. Gudi R, Suber J, Brown R, Johnson BM, Vasu C. Pretreatment with Yeast-derived complex dietary polysaccharides suppresses gut inflammation, alters the microbiota composition, and increases immune regulatory short-chain fatty acid production in C57BL/6 mice. J Nutr. 2020;150:1291-302.

\section{Publisher's Note}

Springer Nature remains neutral with regard to jurisdictional claims in published maps and institutional affiliations.

\section{Submit your manuscript to a SpringerOpen ${ }^{\circ}$ journal and benefit from:}

- Convenient online submission

- Rigorous peer review

- Open access: articles freely available online

- High visibility within the field

- Retaining the copyright to your article

Submit your next manuscript at $\boldsymbol{\nabla}$ springeropen.com 